\title{
Monte Carlo estimation of radiation dose in organs of female and male adult phantoms due to FDG-F18 absorbed in the lungs
}

\author{
Walmir Belinato ${ }^{1,2 a}$, William S. Santos, ${ }^{2}$ Rogério M. V. Silva, ${ }^{2}$ Divanizia N. Souza ${ }^{2}$ \\ ${ }_{2}^{1}$ Federal Institute of Bahia - IFBA, Vitória da Conquista, Bahia, Brazil \\ ${ }^{2}$ Department of Physics, Federal University of Sergipe - UFS, São Cristóvão,Sergipe, Brazil
}

\begin{abstract}
The determination of dose conversion factors ( $\mathrm{S}$ values) for the radionuclide fluorodeoxyglucose $\left({ }^{18} \mathrm{~F}-\mathrm{FDG}\right)$ absorbed in the lungs during a positron emission tomography (PET) procedure was calculated using the Monte Carlo method (MCNPX version 2.7.0). For the obtained dose conversion factors of interest, it was considered a uniform absorption of radiopharmaceutical by the lung of a healthy adult human. The spectrum of fluorine was introduced in the input data file for the simulation. The simulation took place in two adult phantoms of both sexes, based on polygon mesh surfaces called FASH and MASH with anatomy and posture according to ICRP 89 . The S values for the 22 internal organs/tissues, chosen from ICRP No. 110, for the FASH and MASH phantoms were compared with the results obtained from a MIRD V phantoms called ADAM and EVA used by the Committee on Medical Internal Radiation Dose (MIRD). We observed variation of more than $100 \%$ in $\mathrm{S}$ values due to structural anatomical differences in the internal organs of the MASH and FASH phantoms compared to the mathematical phantom.
\end{abstract}

\section{Introduction}

According to estimates from GLOBOCAN 2011, lung cancer has been among the more reported cancers in both men and women in the world, including new cases diagnosed in developed and developing countries [1]. The combination of positron emission tomography (PET) with computed tomography (CT) provides a major improvement in diagnosis and tumor staging, identification and tracking of disseminated disease, improving the planning of radiotherapy, and monitoring the effects of chemotherapy and radiation therapy in patients [2]. For the diagnosis of cancer by PET, [F-18] fluorodeoxyglucose (FDG) is administered through intravenous injection.

To better understand the behaviors of a system, models of physical processes that occur in the system and that simulate a real situation are developed. There are different simulation methods, among which we highlight the Monte Carlo method, based on systematic sampling of random variables. The package MCNPx (Monte Carlo N-Particle) [3] is an extension of MCNP that simulates

\footnotetext{
${ }^{\mathrm{a}}$ Corresponding author: wbfisica@gmail.com
} 
nuclear processes. In medical physics the application is specifically in dosimetry and radiation shielding, reproduction of fission and fusion reactor designs [4].

The Medical Internal Radiation Dose (MIRD) phantoms called ADAM and EVA [5] are also known as mathematical phantoms, and were produced by Fisher and Snyder in 1967 [6]. The organs of this type of phantom are described by mathematical equations, which make them a limited and simplified representation of the actual structure of the human body [7].

The implementation of a routine monitoring program for the calculation of internal doses related to the incorporation of ${ }^{18} \mathrm{~F}$ is difficult, mainly due to its short physical half-life of $1.83 \mathrm{~h} \mathrm{[8].} \mathrm{Therefore,}$ the use of computer calculations is justified in order to know the doses absorbed by patients undergoing PET examinations. In this study we considered the ${ }^{18} \mathrm{~F}$ as a source uniformly distributed in the lungs of the FASH (Female Adult meSH) and MASH (Male Adult meSH) phantoms in order to estimate the determination of the dose conversion factors ( $\mathrm{S}$ values) for 22 internal target organs/tissues of each phantom. S values are directly proportional to the absorbed dose in each target organ. For the estimation, ICRP110, published by the International Commission on Radiological Protection [9], was considered. The results were compared with those determined by the Internal Dose Assessment System (RADAR) [10].

\section{Materials and Methods}

Representative male and female adult human phantoms called FASH and MASH developed by Cassola et al. [6] were used with FDG uniformly distributed in the lungs. Figure 1 shows the placement of the organs contained in the phantoms, with the lungs indicated by arrows.

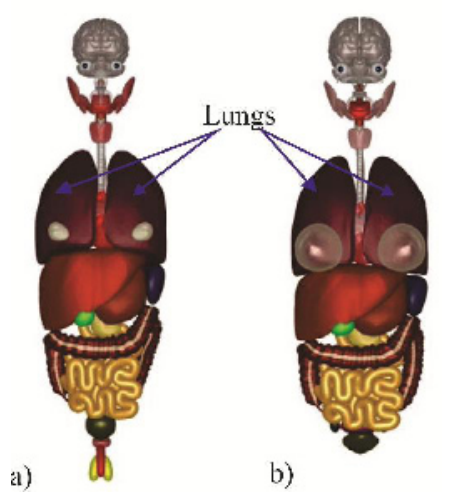

Figure 1. Internal organs in phantoms MASH a) and FASH b) with lungs indicated by arrows. Adapted from [6]

The phantoms' lungs have distinct volumes and consequently different masses. For the ${ }^{18} \mathrm{~F}$, $96.73 \%$ of the emissions are positrons. Its beta energy spectrum [11] is entered into the input file for the code MCNPX. Thereby, we are considering positrons being transported by the source as well as producing annihilation photons.

The absorbed dose rate per unit activity in target organs $\left(\mathrm{r}_{\mathrm{T}}\right)$ due to source organs $\left(\mathrm{r}_{\mathrm{s}}\right)$ is standardized in MIRD Pamphlet 21 [12] according to Equation (1):

$$
\dot{D}\left(r_{T}, t\right)=\sum_{r S} A_{\left(r_{S}, t\right)} S_{\left(r_{T} \leftarrow r_{S}, t\right)}
$$

$A_{\left(r_{S}, t\right)}$ is the radionuclide activity in the organ source and $S_{\left(r_{T} \leftarrow r_{S}, t\right)}$ the $\mathrm{S}$ value described by Equation (2): 


$$
S_{\left(r_{T} \leftarrow r_{S}, t\right)}=\sum_{i} \frac{\Delta_{i} \varnothing\left(r_{T} \leftarrow r_{S}, E_{i}, t\right)}{M_{\left(r_{T}, t\right)}}
$$

Here $\Delta_{\mathrm{i}}$ and $\emptyset\left(r_{T} \leftarrow r_{S}, E_{i}, t\right)$ are the energies deposited by the radionuclide and the fractions of the energy absorbed by the target organs respectively.

The phantoms were placed in a room with dimensions of $4.2 \times 4.2 \times 2.2 \mathrm{~m}$ on a carbon fiber table, approaching the real situation in a PET room.

\section{Results and discussion}

Table 1 presents the $\mathrm{S}$ values obtained with the MASH and FASH phantoms and the $\mathrm{S}$ values provided by the RADAR system.

Table 1. S values obtained with the MASH and FASH phantoms and values calculated by RADAR system (mGy/MBq.s)

\begin{tabular}{lcccc}
\hline \multicolumn{1}{c}{ Organ } & FASH & $\begin{array}{c}\text { EVA } \\
\text { RADAR }\end{array}$ & MASH & $\begin{array}{c}\text { ADAM } \\
\text { RADAR }\end{array}$ \\
\hline Adrenals & $2.79 \mathrm{E}-06(2.89 \%)$ & $2.13 \mathrm{E}-06$ & $2.66 \mathrm{E}-06(2.86 \%)$ & $1.74 \mathrm{E}-06$ \\
Brain & $1.82 \mathrm{E}-07(1.21 \%)$ & $9.98 \mathrm{E}-08$ & $1.38 \mathrm{E}-07(1.30 \%)$ & $9.70 \mathrm{E}-08$ \\
Breasts & $2.00 \mathrm{E}-06(0.75 \%)$ & $1.99 \mathrm{E}-06$ & $2.05 \mathrm{E}-06(3.22 \%)$ & $1.74 \mathrm{E}-06$ \\
Large intestine & $2.43 \mathrm{E}-07(1.69 \%)$ & $2.36 \mathrm{E}-07$ & $1.87 \mathrm{E}-07(1.88 \%)$ & $5.54 \mathrm{E}-07$ \\
Gallbladder wall & $1.36 \mathrm{E}-07(6.50 \%)$ & $6.78 \mathrm{E}-07$ & $1.07 \mathrm{E}-07(6.58 \%)$ & $5.54 \mathrm{E}-07$ \\
Small intestine & $2.04 \mathrm{E}-07(1.46 \%)$ & $1.94 \mathrm{E}-07$ & $1.63 \mathrm{E}-07(1.54 \%)$ & $1.40 \mathrm{E}-07$ \\
Stomach & $1.40 \mathrm{E}-06(1.22 \%)$ & $1.15 \mathrm{E}-06$ & $1.11 \mathrm{E}-06(1.31 \%)$ & $8.23 \mathrm{E}-07$ \\
Heart wall & $6.00 \mathrm{E}-06(0.49 \%)$ & $3.92 \mathrm{E}-06$ & $5.61 \mathrm{E}-06(0.46 \%)$ & $3.00 \mathrm{E}-06$ \\
Kidneys & $1.64 \mathrm{E}-06(0.85 \%)$ & $7.25 \mathrm{E}-07$ & $1.56 \mathrm{E}-06(0.82 \%)$ & $5.08 \mathrm{E}-07$ \\
Liver & $1.93 \mathrm{E}-06(0.37 \%)$ & $1.88 \mathrm{E}-06$ & $1.51 \mathrm{E}-06(0.37 \%)$ & $1.38 \mathrm{E}-06$ \\
Lungs & $4.90 \mathrm{E}-05(0.06 \%)$ & $5.92 \mathrm{E}-05$ & $3.96 \mathrm{E}-05(0.06 \%)$ & $4.69 \mathrm{E}-05$ \\
Muscle & $9.10 \mathrm{E}-07(0.14 \%)$ & $9.02 \mathrm{E}-07$ & $7.68 \mathrm{E}-07(0.12 \%)$ & $6.80 \mathrm{E}-07$ \\
Ovaries/testes & $7.58 \mathrm{E}-0(17.16 \%)$ & $9.74 \mathrm{E}-08$ & $1.03 \mathrm{E}-08(24.44 \%)$ & $7.62 \mathrm{E}-08$ \\
Pancreas & $7.84 \mathrm{E}-07(1.75 \%)$ & $1.61 \mathrm{E}-06$ & $6.43 \mathrm{E}-07(1.76 \%)$ & $1.23 \mathrm{E}-06$ \\
Marrow & $1.63 \mathrm{E}-07(2.10 \%)$ & $9.89 \mathrm{E}-07$ & $1.14 \mathrm{E}-07(1.93 \%)$ & $8.85 \mathrm{E}-07$ \\
Bone surfaces & $9.24 \mathrm{E}-07(0.32 \%)$ & $8.53 \mathrm{E}-07$ & $6.86 \mathrm{E}-07(0.31 \%)$ & $6.64 \mathrm{E}-07$ \\
Skin & $4.55 \mathrm{E}-07(0.52 \%)$ & $4.46 \mathrm{E}-07$ & $3.63 \mathrm{E}-07(0.48 \%)$ & $3.48 \mathrm{E}-07$ \\
Spleen & $2.33 \mathrm{E}-06(1.08 \%)$ & $1.57 \mathrm{E}-06$ & $1.93 \mathrm{E}-06(1.09 \%)$ & $1.14 \mathrm{E}-06$ \\
Thymus & $5.65 \mathrm{E}-06(1.74 \%)$ & $2.45 \mathrm{E}-06$ & $4.74 \mathrm{E}-06(1.69 \%)$ & $2.06 \mathrm{E}-06$ \\
Thyroid & $3.01 \mathrm{E}-06(2.56 \%)$ & $8.28 \mathrm{E}-07$ & $2.60 \mathrm{E}-06(2.52 \%)$ & $6.65 \mathrm{E}-07$ \\
Urine bladder wall & $5.22 \mathrm{E}-08(9.68 \%)$ & $5.05 \mathrm{E}-08$ & $4.66 \mathrm{E}-08(9.15 \%)$ & $2.71 \mathrm{E}-08$ \\
Uterus/prostate & $8.35 \mathrm{E}-08(5.84 \%)$ & $8.55 \mathrm{E}-08$ & $3.79 \mathrm{E}-0(19.57 \%)$ & $5.88 \mathrm{E}-08$ \\
\hline
\end{tabular}

Observing the results in Table 1, variations between $\mathrm{S}$ values due to the different anatomical structures of the internal organs can be perceived. The difference between the masses of the FASH and MASH lungs is $250 \mathrm{~g}$. The density is a parameter to be declared in the input file, and is equal to $0.26 \mathrm{~g} / \mathrm{cm}^{3}$ for both phantoms; therefore there is a difference in volume of approximately $950 \mathrm{~cm}^{3}$ among the sources' organs, producing a different distribution of the source inside the body and, consequently, in the different target organs irradiated.

Figure 2 shows the difference, as a percentage, between the $\mathrm{S}$ values obtained and those published by the RADAR system using a MIRD V phantoms. It can be seen that the $\mathrm{S}$ values of organs like kidneys, thymus, and thyroid show percentage changes of more than $100 \%$ in relation to those obtained by MIRD. 
The MIRD V phantoms represent an adult male and female "reference". Besides using the geometric equations to represent the body and its internal organs, only one model is available for any individual category, so that the dose estimates calculated using this approach will contain significant uncertainties [13]. Considering the different geometries of the organs contained in the MASH and FASH phantoms, as well as the differences in their lungs that were used as source organs in this study, variations in $\mathrm{S}$ values are justifiable.

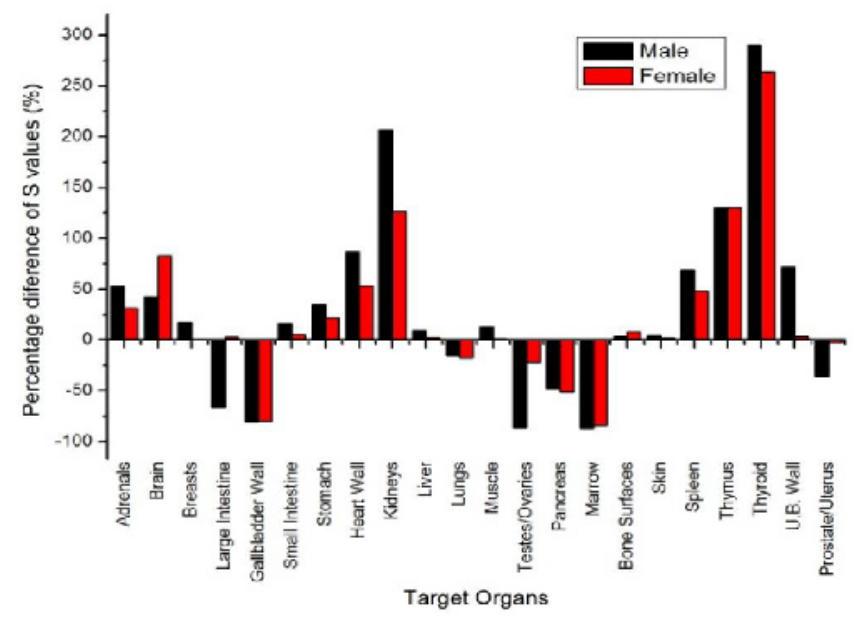

Figure 2. Percentage diference in results with RADAR S-values

\section{Conclusions}

The results for the 22 target organs in the FASH and MASH phantoms showed variations in $\mathrm{S}$ values when compared with those determined by the Internal Dose Assessment System (RADAR). These variances result from the structural anatomical differences in the phantoms studied and in significant changes in the lungs in relation to their sizes in both phantoms.. Whereas there is a proportionality between absorbed dose and $\mathrm{S}$ values, the structural differences of phantoms will result in different dose values.

\section{References}

1. D.V.M. Ahmedin Jemal, et al. CA Cancer J Clin. 61, 69-90 (2011).

2. T. Beyer, et al., J. Nucl. Med. 41, 1369-1379 (2000).

3. D.B. Pelowitz. MCNPX User's Manual, Version 2.7.0. (Report LA-CP-11-00438, Los Alamos National Laboratory, 2011).

4. MCNPx home page. http://mcnpx.lanl.gov. (Last accessed January 2013).

5. $\quad$ R. Kramer et al.. Health Phys. 43, 932-935 (1982).

6. H.L. Fisher, W.S. Snyder. Report ORNL-4168, (Oak Ridge, TN, USA, 1967).

7. V.F. Cassola, V.J de Melo Lima, R. Kramer, H.J. Khoury. Phys. Med. Biol. 55; 133-162 (2009).

8. D. Delacroix, J.P. Guerre, P. Leblanc, C. Hickman. Radionuclide and radiation protection data handbook 2002. Rad. Prot. Dosim. 98 (1), 5-6 (2002).

9. ICRP. Adult Reference Computational Phantoms. ICRP Publication 110. Ann. ICRP 39(2) (2003).

10. M.G. Stabin, J.A. Siegel. Physical. Health Phys. 85(3), 294-310 (2003).

11. K.F. Eckerman, R.J. Westfall, J.C. Ryman, M. Cristy. Health Phys. 67(4), 338-345 (1994).

12. W.E. Bloch, K.F. Eckerman, G. Sgorus, S.R. Thomas. J. Nucl. Med. 50, 477-484 (2009).

13. M.G. Stabin, G.D. Flux. Biomed Imaging Interv. J. 3(2), e28 (2007). 